\title{
Application of Correlative Fluorescence and Electron Microscopy for Morphological Characterization of Individual Transfected Pancreas and Biliary Epithelial cells
}

B. Q. Huang, X. M. Chen, E. W. Krueger, L. S. Orlichenko, N. F. LaRusso and M. A. McNiven

The Center for Basic Research in Digestive Diseases, Department of Biochemistry and Molecular Biology, Mayo Clinic, Rochester, MN 55905

Precise identification of transfected cells and localization of their specific intracellular biomolecules is a crucial step in understanding cellular functions. The application of electron microscopy in conjunction with fluorescence labeling has proved to be advantageous for high-resolution structural studies [1] and is important for understanding the phenotype of mutant cells [2]. However, localization of individual mutant cells in a cultured cell population at low-transfection rate and detection of antigen-antibody binding in a small number of single mutant cells is technically difficult. Recently, FluoroNanogold as a new dual immunolabeling probe has been successfully used for correlative immunofluorescent and immunoelectron microscopy to detect individual antigens in selected specimens [3] and, thus, is a useful probe to address the aforementioned challenge. Here we report that two techniques of correlative microscopy have been developed for morphological characterization of single transfected pancreatic and biliary epithelial cells.

Firstly, a technique was developed for precise localization of caveolin-1 (cav-1) transfected human cultured pancreatic cells for electron microscopic analysis using special etched coverslips. Human Panc-1 cells were grown on carbon-coated, etched coverslips and transfected with modified cav-1 tagged with GFP and then stimulated with EGF. Phase and fluorescent images were captured to localize transfected and nontransfected cells through finder grids on the etched coverslip (FIG. 1, 2). The cells were then sectioned and loaded on the copper grid (FIG. 3, 4). The number of caveolae in the transfected cells (FIG. 5) was found to be substantially reduced below that of the nontransfected cells (FIG. 6). This technique has been successfully applied to unambiguously identify and morphologically characterize a variety of single mutant cells including Dyn2. Secondly, we applied correlative microscopy to detect special antigen-antibody binding in cultured human biliary epithelial cells infected by Cryptosporidium parvum with FluoroNanogold. Anti-AQP1 antibody labeling and AQP1-GFP fluorescence showed that AQP1 is accumulated at the attachment site between C. parvum and biliary epithelial cells (FIG. 7, 8). Correlative immuno-EM detected accumulation of AQP1 at the protruded membrane of the host cell and parasite-host cell interface [FIG. 9, 10]. Using these techniques we are able to precisely localize the specific single mutant cells and morphologically characterize the parasite invasion events and primary cilia in the biliary epithelial cells in situ effectively using both light and electron microscopy. 


\section{References}

[1] F.M. Boisvert et al., J. Cell Biol. 148 (2000) 283-292.

[2] R. S. Polishuk et al., J. Cell Biol. 148 (2000) 45-58.

[3] T. Takizawa and J.M.Robinson J. Histoch. \& Cytochem. 51 (2003) 707-714.
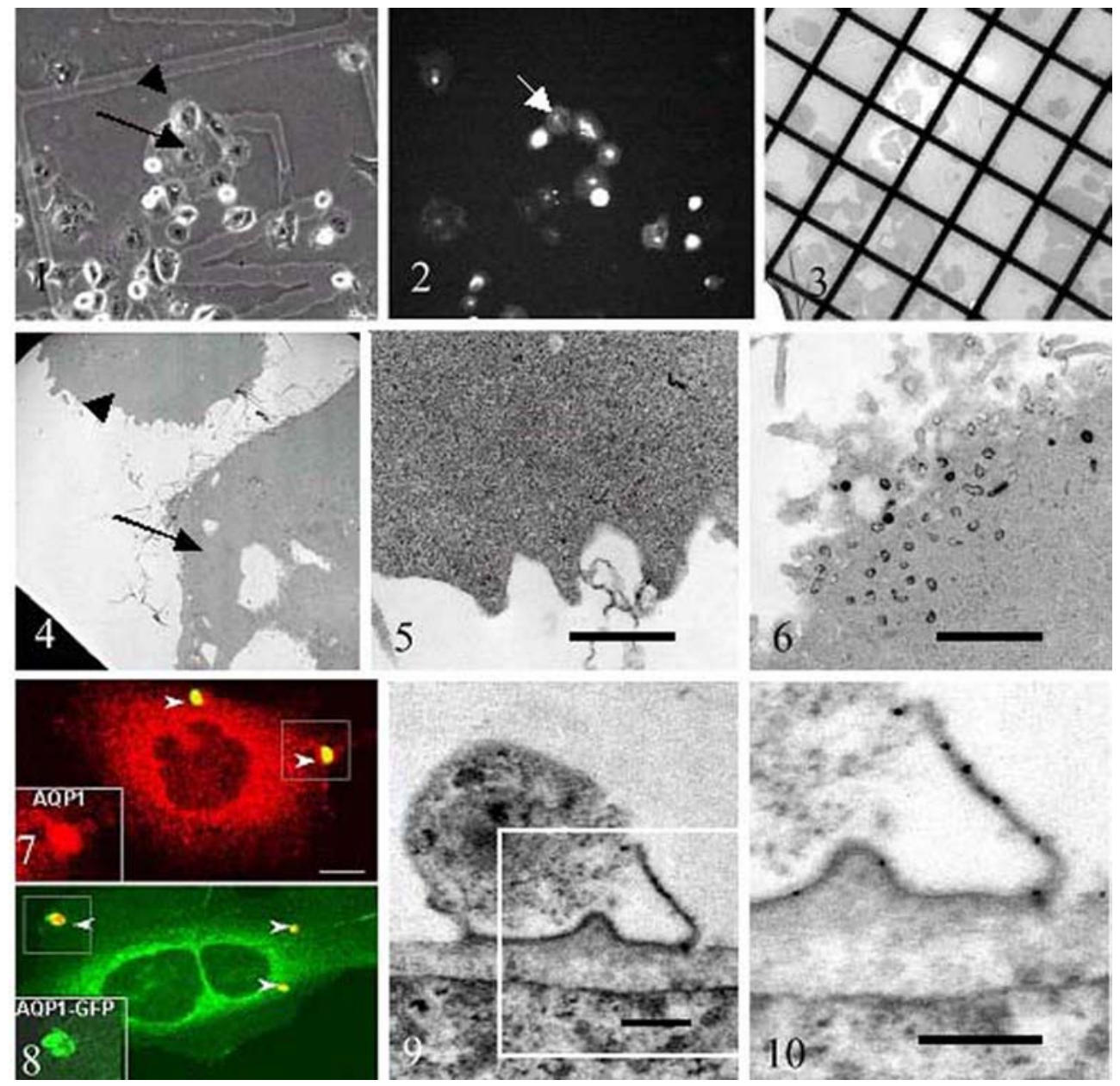

FIG.1 Phase image of cav-1 human Panc1 cells including transfected (arrowhead) and non-transfected cells (arrow). FIG. 2 GFP-fluorescence of transfected cells (arrowhead). FIG.3 EM image of the same group of cells as shown in FIG. 1. FIG. 4 EM image of a transfected (arrowhead) and a non-transfected cell (arrow). FIG. 5. Few caveolae were found in transfected EGF-stimulated cells. Bar $=1 \mu \mathrm{m}$. FIG. 6 Abundant caveolae in the peripheral region of the non-transfected EGF-stimulated cell. FIG. 7 Anti-AQP1 fluorescence shows the accumulation of AQP-1 at the parasite-host cell interface in the human biliary epithelial cell (arrowheads). Bar $=10 \mu \mathrm{m}$. FIG. 8. AQP1-GFP fluorescence shows strong fluorescence at the parasite-host cell interface (arrowheads). Bar $=10 \mu \mathrm{m}$. FIG. 9 TEM image of attachment of $C$. parvum to the biliary epithelial cell. Bar $=1 \mu \mathrm{m}$. FIG. 10 Immuno-EM shows gold particles at the protruded membrane of host cell and parasite-host cell interface. Bar $=1 \mu \mathrm{m}$. 